\title{
A Hierarchical Illumination Algorithm for Surfaces with Glossy Reflection
}

\author{
Larry Aupperle Pat Hanrahan \\ Department of Computer Science \\ Princeton University
}

\begin{abstract}
We develop a radiance formulation for discrete three point transport, and a new measure and description of reflectance: area reflectance. This formulation and associated reflectance allow an estimate of error in the computation of radiance across triples of surface elements, and lead directly to a hierarchical refinement algorithm for global illumination.

We have implemented and analyzed this algorithm over surfaces exhibiting glossy specular and diffuse reflection. Theoretical growth in light transport computation is shown to be $O\left(n+k^{3}\right)$ for sufficient refinement, where $n$ is the number of elements at the finest level of subdivision over an environment consisting of $k$ input polygonal patches - this growth is exhibited in experimental trials. Naive application of three point transport would require computation over $O\left(n^{3}\right)$ element-triple interactions.
\end{abstract}

CR Categories and Subject Descriptors: I.3.7 [Computer Graphics]: Three-Dimensional Graphics and Realism.

Key Words: adaptive meshing, global illumination, radiosity, ray tracing.

\section{Introduction}

A major open problem in image synthesis is the efficient solution of the rendering equation. Radiosity methods have been quite successful over environments containing surfaces that exhibit only diffuse reflection. Unfortunately, very few materials are purely Lambertian reflectors, and efficient solution techniques have not yet been developed for more general specular or glossy reflection functions.

The rendering equation is an integral equation, and the solutions to complicated integral equations are generally obtained using either Monte Carlo or finite element techniques. Monte Carlo algorithms sometimes go under the name of distributed or stochastic ray tracing and are the most commonly employed in computer graphics (e.g. see $[4,5,9,12,16])$. Monte Carlo techniques have the advantage that they are easy to implement and can be used for complicated geometries and reflection functions. Unfortunately, their disadvantage is that they are notoriously inefficient. The second approach, the finite element method, has been very successfully applied to the rendering equation under the radiosity assumption, but has only begun to be employed in the general case, and with limited success. For example, Immel et al. [8] discretized radiance into a lattice of cubical environment maps, and solved the resulting system. More recently, Sillion et al. [13] used a mesh of spherical harmonic functions to represent radiance, and solved the resulting system using a shooting algorithm.

Permission to copy without fee all or part of this material is granted provided that the copies are not made or distributed for direct commercial advantage, the ACM copyright notice and the title of the publication and its date appear, and notice is given that copying is by permission of the Association for Computing Machinery. To copy otherwise, or to republish, requires a fee and/or specific permission. (C)1993 ACM-0-89791-601-8/93/008 ...\$1.50
There are many ways to parameterize the rendering equation, and each leads to a different choice of basis functions. In the transport theory community two techniques are common: directional subdivision (the method of discrete ordinates or $S_{N}$ ), and spherical harmonics $\left(P_{N}\right)$. These two techniques roughly correspond to the methods of Immel et al. and Sillion et al., although many interesting variations are possible. Our approach is somewhat different, and based on Kajiya's original formulation of the rendering equation [9]. Under this formulation, the rendering equation is expressed in terms of three point transport. That is, the kernel of the integral expresses the transport of light from a point on the source to a point on the receiver, via a point on a reflector. Given this formulation, the three point rendering equation can be discretized over pairs of elements to form a linear system of equations. Solving this system yields the radiance transported between elements. Note that this approach is very similar to the radiosity formulation.

The problem with finite element methods is that the matrix of interactions is very large for interesting environments. For a given environment of $k$ input polygonal patches containing $n$ elements at the finest level of refinement, the three point discretization that we are proposing generates an $n^{3}$ matrix of interactions. However, in this paper we show that we can accurately approximate the $n^{3}$ reflectance matrix with $O\left(n+k^{3}\right)$ blocks, in a way very similar to our recent hierarchical radiosity algorithm [7]. In that paper we showed how the $n^{2}$ form factor matrix could be approximated with $O\left(n+k^{2}\right)$ blocks, resulting in a very efficient algorithm in both space and time. Although the results presented in this paper are preliminary, we believe a hierarchical finite element approach along these lines will ultimately lead to a fast, efficient algorithm.

In the following section we describe our application of the finite element element method to the three point rendering equation, yielding a radiance formulation for discrete transport. In Section 3 we present a simple adaptive refinement algorithm for computation over this formulation, and the iterative solution technique employed for the actual calculation of transport. In Section 4 we discuss our implementation of the algorithm over glossy reflection, and in Section 5 we present some experiments and results. An appendix to this paper contains details of our error analysis for discrete transport under the glossy model.

\section{Discrete Three Point Transport}

The algorithm presented in this paper operates through two functions: refinement of the environment to form a hierarchy of discrete interactions, patches and elements, and the actual computation of illumination over this hierarchy.

In this section we develop the basis for both discretization and transport. We derive a radiance formulation for three point transport, and a new measure and description of reflectance, area reflectance. This radiance formulation and associated reflectance provide a natural criterion for discretization under illumination and reflection, and allow both the computation of radiance across triples of individual surface elements, and the expression and computation of all light transport over all surfaces. 


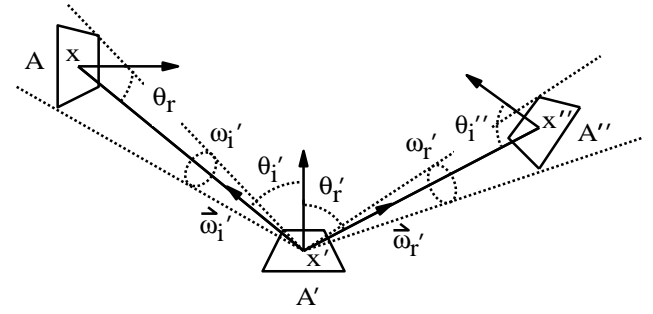

Figure 1: Geometry of Reflection

\subsection{A Radiance Formulation for Three Point Transport}

When computing and imaging illumination within an environment, we are interested in the transport of light from surface to surface it is this interaction of surfaces that characterizes illumination, in the absence of participatory media. Reflection within an environment may thus be naturally expressed over triples of surfaces. Consider surfaces $A, A^{\prime}$, and $A^{\prime \prime}$ (Figure 1) - we will examine the transport of light incident at $A^{\prime}$ originating at $A$ and reflected toward $A^{\prime \prime}$.

Let $\omega_{i}^{\prime}$ and $\omega_{r}^{\prime}$ be the solid angles subtended at point $x^{\prime}$ by $A$ and $A^{\prime \prime}$, respectively. Consider differential solid angles at $\vec{\omega}_{i}^{\prime}$ and $\vec{\omega}_{r}^{\prime}-$ by definition of the bidirectional reflectance-distribution function (BRDF), $f_{r}$ [11], the radiance $L\left(\vec{\omega}_{r}^{\prime}\right)$ along $\vec{\omega}_{r}^{\prime}$ due to illumination through solid angle $\omega_{i}^{\prime}$ is:

$$
L\left(\vec{\omega}_{r}^{\prime}\right)=\int_{\omega_{i}^{\prime}} f_{r}\left(\vec{\omega}_{i}^{\prime}, \vec{\omega}_{r}^{\prime}\right) L\left(\vec{\omega}_{i}^{\prime}\right) \cos \theta_{i}^{\prime} d \omega_{i}^{\prime}
$$

Integrating this expression over $\omega_{r}^{\prime}$, and introducing $\cos \theta_{r}^{\prime}$, we have:

$$
\begin{aligned}
& \int_{\omega_{r}^{\prime}} L\left(\vec{\omega}_{r}^{\prime}\right) \cos \theta_{r}^{\prime} d \omega_{r}^{\prime}= \\
& \quad \int_{\omega_{r}^{\prime}} \int_{\omega_{i}^{\prime}} f_{r}\left(\vec{\omega}_{i}^{\prime}, \vec{\omega}_{r}^{\prime}\right) L\left(\vec{\omega}_{i}^{\prime}\right) \cos \theta_{i}^{\prime} \cos \theta_{r}^{\prime} d \omega_{i}^{\prime} d \omega_{r}^{\prime}
\end{aligned}
$$

We may then reparameterize over $A$ and $A^{\prime \prime}$ to yield:

$$
\begin{aligned}
\int_{A^{\prime \prime}} L\left(x^{\prime}, x^{\prime \prime}\right) G\left(x^{\prime}, x^{\prime \prime}\right) d x^{\prime \prime}= \\
\quad \int_{A} \int_{A^{\prime \prime}} f_{r}\left(x, x^{\prime}, x^{\prime \prime}\right) L\left(x, x^{\prime}\right) G\left(x, x^{\prime}\right) G\left(x^{\prime}, x^{\prime \prime}\right) d x^{\prime \prime} d x
\end{aligned}
$$

where

$$
G\left(x, x^{\prime}\right)=\frac{\cos \theta_{r} \cos \theta_{i}^{\prime}}{\left|x-x^{\prime}\right|^{2}} v\left(x, x^{\prime}\right)
$$

where $v\left(x, x^{\prime}\right)$ is 1 if points $x, x^{\prime}$, are mutually visible, and 0 otherwise. Note that $G$ is very similar to a differential form factor.

We integrate over $A^{\prime}$, thus introducing all three areas into the formulation:

$$
\begin{aligned}
\int_{A^{\prime}} & \int_{A^{\prime \prime}} L\left(x^{\prime}, x^{\prime \prime}\right) G\left(x^{\prime}, x^{\prime \prime}\right) d x^{\prime \prime} d x^{\prime}= \\
& \int_{A} \int_{A^{\prime}} \int_{A^{\prime \prime}} f_{r}\left(x, x^{\prime}, x^{\prime \prime}\right) L\left(x, x^{\prime}\right) G\left(x, x^{\prime}\right) G\left(x^{\prime}, x^{\prime \prime}\right) d x^{\prime \prime} d x^{\prime} d x
\end{aligned}
$$

We may now rewrite the equation in discrete form. Let $A_{j}$ and $A_{k}$ be subareas of $A^{\prime}$ and $A^{\prime \prime}$ such that $L\left(x^{\prime}, x^{\prime \prime}\right)$ is nearly constant over their surfaces. The left side of equation (1) may then be rewritten, bringing radiance out of the integral as $L_{j k}$ :

$$
L_{j k} \int_{A_{j}} \int_{A_{k}} G\left(x^{\prime}, x^{\prime \prime}\right) d x^{\prime \prime} d x^{\prime}=\pi L_{j k} A_{j} F_{j k}
$$

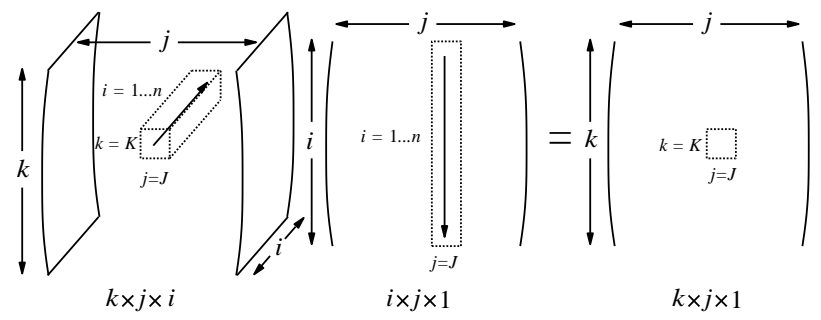

Figure 2: A Reflection Product

by definition of the diffuse form factor, $F_{j k}$.

We may similarly discretize $A$ as $A_{i}$, and rewrite the right side of equation (1) as:

$$
\begin{gathered}
L_{i j} \int_{A_{i}} \int_{A_{j}} \int_{A_{k}} f_{r}\left(x, x^{\prime}, x^{\prime \prime}\right) G\left(x, x^{\prime}\right) G\left(x^{\prime}, x^{\prime \prime}\right) d x^{\prime \prime} d x^{\prime} d x= \\
\sum_{i} \pi L_{i j} A_{i} F_{i j} R_{i j k}
\end{gathered}
$$

where $R_{i j k}$ is defined such that

$$
\begin{aligned}
& \pi A_{i} F_{i j} R_{i j k}= \\
& \qquad \int_{A_{i}} \int_{A_{j}} \int_{A_{k}} f_{r}\left(x, x^{\prime}, x^{\prime \prime}\right) G\left(x, x^{\prime}\right) G\left(x^{\prime}, x^{\prime \prime}\right) d x^{\prime \prime} d x^{\prime} d x
\end{aligned}
$$

Note that, by the symmetry of $f_{r}$ and $G$ :

$$
A_{i} F_{i j} R_{i j k}=A_{k} F_{k j} R_{k j i}
$$

We thus have:

$$
\begin{aligned}
\pi L_{j k} A_{j} F_{j k} & =\pi \sum_{i} L_{i j} A_{k} F_{k j} R_{k j i} \\
& =\pi \sum_{i} L_{i j} A_{j} F_{j k} R_{k j i}
\end{aligned}
$$

by the reciprocity of form factors, and thus:

$$
L_{j k}=\sum_{i} L_{i j} R_{k j i}
$$

The three dimensional character of $R_{k j i}$ over indices $i, j, k$ leads naturally to a three dimensional matrix formulation for the above system. Consider a product over an $n \times n \times n R_{k j i}$ "matrix" and an $n \times n \times 1 L_{i j}$ matrix producing an $n \times n \times 1$ matrix of reflected radiances, as shown in Figure 2. Note that the $R_{k j i}$ matrix is of size $O\left(n^{3}\right)$ - the hierarchical method discussed in subsequent sections of this paper addresses more tractable representation of this matrix.

Taking into account emission, we have derived a radiance formulation for three point transport:

$$
L_{j k}=E_{j k}+\sum_{i} L_{i j} R_{k j i}
$$

This formulation states that:

The radiance at Area $j$ in the direction of Area $k$ is equal to the radiance emitted by $j$ in the direction of $k$, plus, for every Area $i$, the radiance at $i$ in the direction of $j$ multiplied by the area reflectance $R_{k j i}$.

Note that equation (2) is very similar to the radiosity formulation:

$$
B_{j}=E_{j}+\rho_{j} \sum_{i} B_{i} F_{j i}
$$




\subsection{Area Reflectance}

The quantity $R_{k j i}$ has a natural and satisfying physical significance - it is an expression of reflectance over areas $A_{i}, A_{j}$, and $A_{k}$.

Consider the fraction of the radiant flux transported from $A_{i}$ incident to $A_{j}$ that is reflected in the direction of area $A_{k}$ :

$$
\frac{\int_{A_{i}} \int_{A_{j}} \int_{A_{k}} f_{r}\left(x, x^{\prime}, x^{\prime \prime}\right) L\left(x, x^{\prime}\right) G\left(x, x^{\prime}\right) G\left(x^{\prime}, x^{\prime \prime}\right) d x^{\prime \prime} d x^{\prime} d x}{\int_{A_{i}} \int_{A_{j}} L\left(x, x^{\prime}\right) G\left(x, x^{\prime}\right) d x^{\prime} d x}
$$

If we assume that incident radiance is uniform and isotropic over both $\omega_{i}^{\prime}$ (as induced by $A_{i}$ ) and $A_{j}$, we may divide through by $L\left(x, x^{\prime}\right)$, yielding:

$$
\begin{aligned}
& \rho\left(A_{i}, A_{j}, A_{k}\right) \equiv \\
& \frac{\int_{A_{i}} \int_{A_{j}} \int_{A_{k}} f_{r}\left(x, x^{\prime}, x^{\prime \prime}\right) G\left(x, x^{\prime}\right) G\left(x^{\prime}, x^{\prime \prime}\right) d x^{\prime \prime} d x^{\prime} d x}{\int_{A_{i}} \int_{A_{j}} G\left(x, x^{\prime}\right) d x^{\prime} d x}
\end{aligned}
$$

We define $\rho\left(A_{i}, A_{j}, A_{k}\right)$ to be area reflectance. Note that area reflectance is similar to biconical reflectance [11], save that it is also integrated over the reflecting surface.

By definition of $R_{i j k}$ :

$$
R_{i j k}=\rho\left(A_{i}, A_{j}, A_{k}\right)
$$

Conservation of energy over reflection, and the reciprocity relation derived for $R_{i j k}$ above, constitute fundamental properties of area reflectance:

1. $\sum_{k} R_{i j k} \leq 1$, for fixed $i, j$.

2. $A_{i} F_{i j} R_{i j k}=A_{k} F_{k j} R_{k j i}$.

where equality is achieved in property 1 over complete enclosures and perfect reflectivity.

\subsection{Evaluation of $R_{k j i}$}

In this section we examine the evaluation of $R_{k j i}$ over given patches $A_{i}, A_{j}, A_{k}$.

Recall:

$$
R_{k j i}=\frac{\int_{A_{k}} \int_{A_{j}} \int_{A_{i}} f_{r}\left(x^{\prime \prime}, x^{\prime}, x\right) G\left(x^{\prime \prime}, x^{\prime}\right) G\left(x^{\prime}, x\right) d x d x^{\prime} d x^{\prime \prime}}{\int_{A_{k}} \int_{A_{j}} G\left(x^{\prime \prime}, x^{\prime}\right) d x^{\prime} d x^{\prime \prime}}
$$

We assume that discrete areas $A_{i}, A_{j}, A_{k}$ are of small enough scale that $f_{r}$ and $G$ are relatively constant over their surfaces. Then:

$$
\begin{aligned}
R_{k j i} & =\frac{S_{k j i} G_{k j} G_{j i} A_{k} A_{j} A_{i}}{G_{k j} A_{k} A_{j}} \\
& =S_{k j i} G_{j i} A_{i}
\end{aligned}
$$

where $S$ is the discretized value of $f_{r}, S_{i j k}=S_{k j i}=S_{x_{k} x_{j} x_{i}}$.

Note that the average value of $G\left(x^{\prime}, x\right)$ over $A_{i}$ and $A_{j}$ is $\pi F_{j i} / A_{i}$ - we thus estimate $G_{j i} A_{i}$ by $\pi F_{j i}$, and compute $R_{k j i}$ as:

$$
R_{k j i}=\pi F_{j i} S_{k j i}
$$

In practice, it will not be possible to compute the exact values of $F_{j i}$ and $S_{k j i}$ over $A_{i}, A_{j}, A_{k}$. We assume that we are able to estimate these values, along with error bounds for each estimation. Let $\Delta F_{j i}$ and $\Delta S_{k j i}$ be error estimates for computed $F_{j i}$ and $S_{k j i}$, respectively. We then have an estimate for area reflectance in the form:

$$
\begin{aligned}
R_{k j i} & =\pi\left(F_{j i}+\Delta F_{j i}\right)\left(S_{k j i}+\Delta S_{k j i}\right) \\
& =\pi\left(F_{j i} S_{k j i}+\Delta F_{j i} S_{k j i}+\Delta S_{k j i} F_{j i}+\Delta F_{j i} \Delta S_{k j i}\right) \\
& \approx \pi\left(F_{j i} S_{k j i}+\Delta F_{j i} S_{k j i}+\Delta S_{k j i} F_{j i}\right)
\end{aligned}
$$

Assuming $\Delta F_{j i}<F_{j i}, \Delta S_{k j i}<S_{k j i}$, we have neglected the last term and estimate the error in $R_{k j i}$ as $\pi\left(\Delta F_{j i} S_{k j i}+\Delta S_{k j i} F_{j i}\right)$.
In general, and as is shown for glossy reflection in Section 4, the accuracy of estimators for $F_{j i}$ and $S_{k j i}$ is dependent on the size of the patches over which reflectance is computed, relative to their distance apart. As relative size decreases, so does error in computation, leading directly to the adaptive refinement strategy for illumination presented in Section 3 below.

\section{Algorithms for Three Point Transport}

\subsection{Introduction}

Recall equation (2):

$$
L_{j k}=E_{j k}+\sum_{i} L_{i j} R_{k j i}
$$

This equation suggests both a solution strategy for radiance under three point transport, and a natural representation for illumination within the solution system.

We may interpret equation (2) as a gathering iteration similar to that employed for radiosity under diffuse reflection: the radiance $L_{j k}$ at patch $A_{j}$ in the direction of patch $A_{k}$ is found by gathering radiances $L_{i j}$ in the direction of $A_{j}$ at patches $A_{i}$. We may solve for transport by gathering radiance for each $L_{j k}$, and successively iterating to capture all significant re-reflection.

We are left with the question of what structure we are gathering over and iterating upon. Note that all illumination is expressed as the radiance at a given patch in the direction of another - it is these patch-patch interactions that form the primary structure within the solution system. All operation is over interactions: both the representation and transport of radiance, and the iteration and solution for illumination.

Consider the following structure:

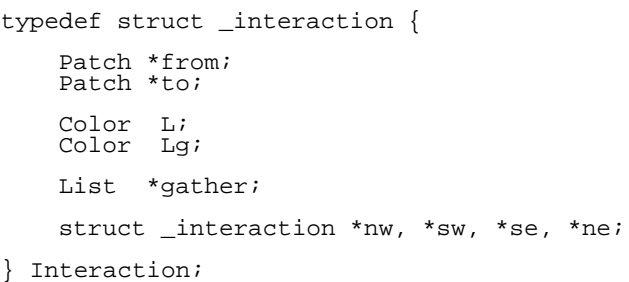

A given interaction $i j$ is defined by two patches $i j->$ from and $i j->t o$, and represents the radiance at from in the direction of to. This radiance is stored within the interaction as attribute $\mathrm{L}$. $\mathrm{Lg}$ is radiance gathered during the current solution iteration from interactions contained in the list gather. Subinteractions nw, sw, se, ne are the children of $i j$, induced by subdivision over either from or to. The structure assumes quadtree refinement, leaving northwest, southwest, southeast, and northeast descendants.

In the following sections we will present an algorithm for the refinement and computation of illumination over a hierarchy of interactions. The algorithm will operate by refining pairs of interactions $i j, j k$ (such that $i j->t o==j k->$ from), to ensure that computed reflectance across the interaction pairs, and associated patch triples, satisfies user specified error bounds. If a given interaction pair $i j, j k$ is satisfactory, the interactions are linked to record that radiance may be gathered from $i j$ to $j k$, otherwise one or both interactions are subdivided and refinement applied to their descendants.

After refinement, a gathering iteration may be carried out, each interaction gathering radiance from interactions to which it has been linked. The gathered radiances are then distributed within each receiving interaction hierarchy, and subsequent iterations computed until satisfactory convergence has been achieved.

Note that, within this system, the eye may be regarded as simply another object with which patches may interact. The radiance along interactions to the eye provides the resulting view. 


\subsection{Adaptive Refinement}

Consider the following procedure:

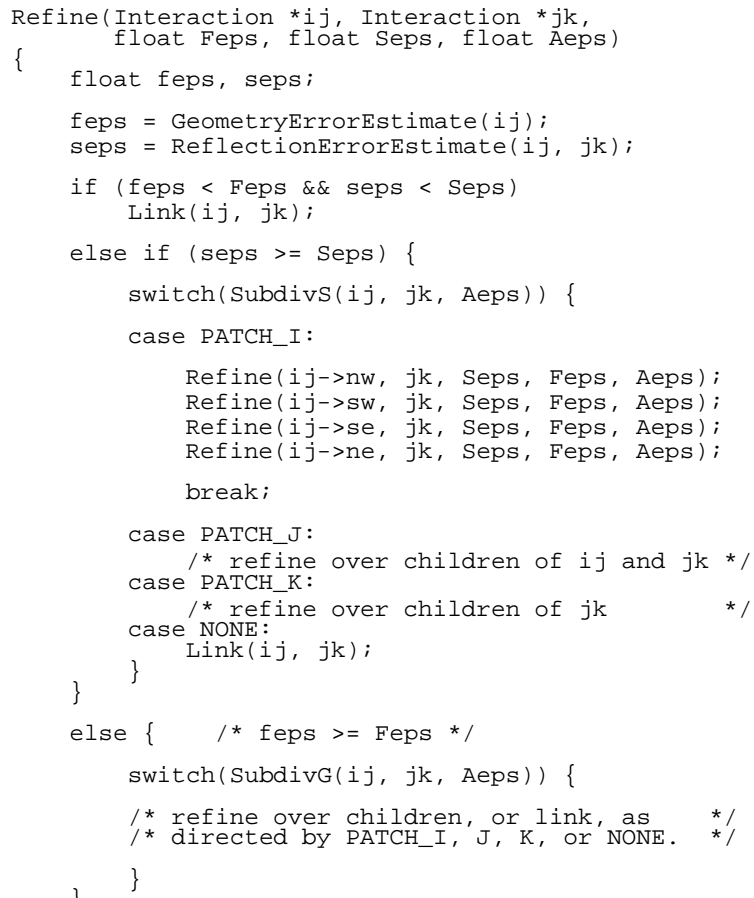

This procedure computes over pairs of interactions, and associated patch triples, subdividing and recursively refining if estimated error exceeds user specified bounds, linking the interactions for gathering if the bounds are satisfied, or if no further subdivision is possible. Feps and Seps are the bounds for geometric and reflection error, respectively; Aeps specifies the minimum area a patch may possess and still be subdivided. GeometryErrorEstimate and ReflectionErrorEstimate provide estimations for $\pi \Delta F_{j i} S_{k j i}$ and $\pi \Delta S_{k j i} F_{j i}$.

SubdivS and Subdivg control refinement for reflection and geometry error, respectively. Both routines select a patch for refinement, subdividing the patch and associated interaction(s) if required. An identifier for the selected patch is returned - if no patch may be subdivided, then NONE is passed back. Note that a given interaction/patch may be refined against many different interactions within the system, and thus may have already been subdivided when selected by a Subdiv routine - in this case, the routine simply returns the proper identifier.

The Subdiv routines should select for refinement patches that are of large size relative to their distance from their partner(s) in the transport triple. Form factor estimation is a convenient criterion for the determination of such patches - a large differential to area form factor $F_{d p q}$ indicates that patch $q$ is of large relative size. Care must be taken in subdivision, however, to ensure that each interaction is always subdivided in the same way for all refinements involving that interaction.

The Subdiv routines thus choose for refinement the patch of size at least Aeps that is of greatest form factor within $i j$ and/or $j k$ that will not induce multiple sets of children over either interaction. If patch $p_{j}$ is of greatest form factor over both $i j$ and $j k$, and of area greater than Aeps, then it is chosen for refinement (Figure 3 at middle). Otherwise, if $p_{j}$ is selected over one interaction, but $p_{i}$ or $p_{k}$ is selected over the other, then the "outside" patch is chosen for refinement. Given two selected outside patches, SubdivS selects the one of greater form factor relative to $p_{j}$; SubdivG selects $p_{i}$ over $p_{k}$, as $p_{k}$ has no direct effect on geometric accuracy. Note, however, that even under SubdivG, if only $p_{j}$ and $p_{k}$ are allowed subdivision, $p_{k}$ will be selected, although with further subdivision the triple will eventually balance sufficiently to allow refinement over $p_{j}$.

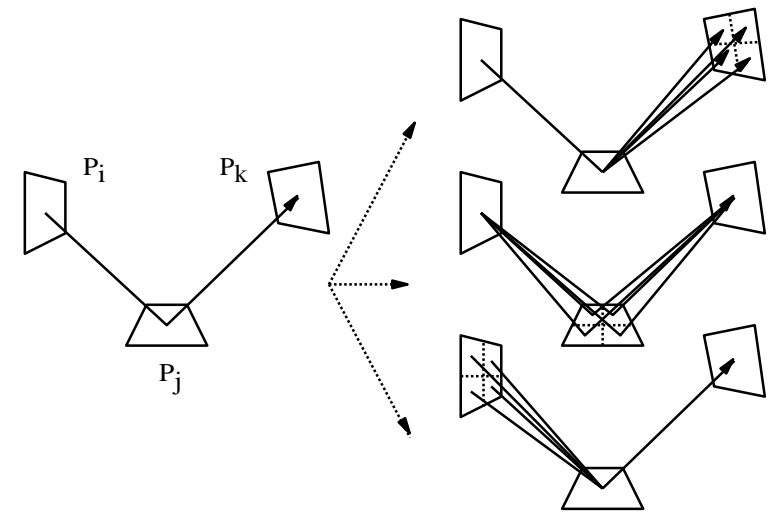

Figure 3: Refinement and Subdivision

\subsection{Gathering Radiance}

Gathering radiance over interactions may be written as a simple procedure:

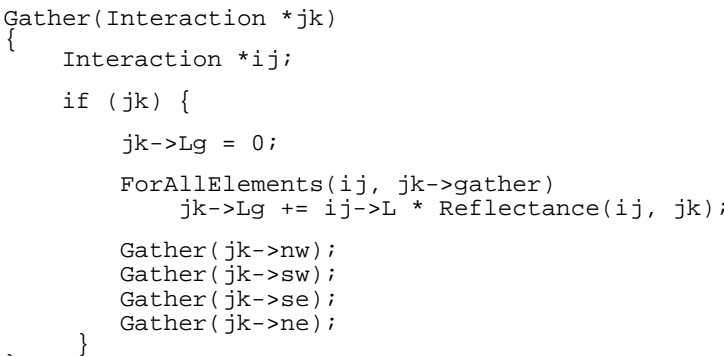

We gather radiance into $j k->L g$ rather than directly into $j k->L$ to avoid the necessity of a push/pull with every invocation of the procedure (see Section 3.4). The solution method is thus simple Jacobi iteration, as opposed to Gauss-Seidel, as the hierarchical structure imposes simultaneous rather than successive displacement.

\subsection{Radiance within a Hierarchy}

A gathering iteration results in received radiance scattered throughout each interaction hierarchy. This gathered radiance must be distributed and accounted for over all ancestors and descendants of each receiving interaction, in order to maintain the consistency and correctness of the hierarchical representation of radiance between patches.

We employ a distribution algorithm similar to that presented in [7] for radiosity over patch/element hierarchies: gathered radiance is "pushed" to the leaf interactions within each hierarchy to ensure propagation to all descendants, and then "pulled" and distributed back up from the leaves through all higher level interactions to their common ancestor at the root. As is shown in [2], radiance may be pushed unchanged within the interaction hierarchy, and area averaged as it is pulled from child to parent.

\section{Application over Glossy Reflection}

In this section we discuss our implementation of the above algorithms over glossy reflection.

\subsection{The Reflection Function}

We employ a highly simplified Torrance-Sparrow [15] model for our glossy reflection function:

$$
f_{g}\left(\vec{\omega}_{i}, \vec{\omega}_{r}\right)=\frac{\kappa+2}{8 \pi} \frac{\cos ^{\kappa} \theta_{m}}{\cos \theta_{i} \cos \theta_{r}} \operatorname{sh}\left(\theta_{i}, \theta_{r}\right)
$$

This function incorporates the facet distribution function $\cos ^{\kappa} \theta_{m}$ developed by Blinn [3], normalized for projected facet area under 


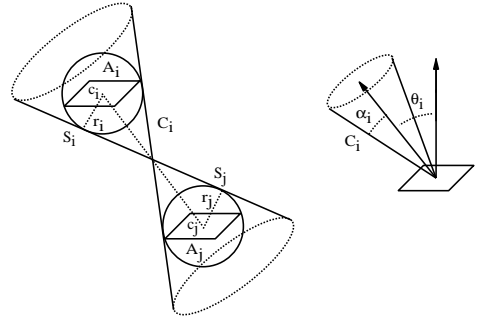

Figure 4: Estimating Cones

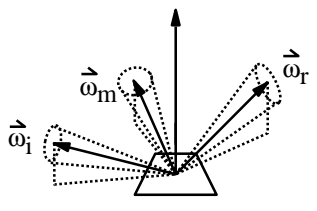

Figure 5: $C_{i}, C_{r}$, and $C_{m}$

[10]. Angle $\theta_{m}$ is that made to the mean surface normal by $\vec{\omega}_{m}$, the microfacet mirror orientation normal lying halfway between $\vec{\omega}_{i}$ and $\vec{\omega}_{r}$.

Function $\operatorname{sh}\left(\theta_{i}, \theta_{r}\right)$ expresses self-shadowing over microfacets - for near specular surfaces, such self-shadowing or masking does not become critical until relatively high $\theta_{i}$ or $\theta_{r}$ [6]. The implemented system thus simply clamps $s h$ from 1 to 0 when $\theta_{i}$ or $\theta_{r}$ exceeds a preset $\theta_{\text {bound }}$ near the horizon. This scheme serves as a crude approximation to the shadowing function; however, a better strategy would be to employ a much fuller tabulation of the function, incorporated into the error analysis presented below. A more complete discussion of shadowing and conservation of energy over $f_{g}$ is presented in [2].

\subsection{Error Estimation}

Recall the general expression for error derived in Section 2.3:

$$
\pi\left(\Delta F_{j i} S_{k j i}+\Delta S_{k j i} F_{j i}\right)
$$

In implementation we have estimated the form factor $F_{j i}$ by $F_{d j i}$, the form factor from a differential area at $A_{j}$ to a disk of area $A_{i}$ centered at $A_{i}$, as was employed in [7]. As discussed in [7], the relative error in this estimate is proportional to the estimate itself. In our implementation we have thus estimated absolute error $\Delta F_{j i}$ as at most proportional to $F_{d j i}^{2}$. A brief discussion of relative and absolute error over hierarchical methods is presented in [2].

We now consider the error estimate $\Delta S_{k j i}$. As discussed in the appendix to this paper, we may compute bounding cones $C_{i}, C_{r}$, and $C_{m}$ over all possible incident, reflected, and mirror orientation directions induced at $A_{j}$ by $A_{i}$ and $A_{k}$ (Figures 4 and 5 - these figures are discussed more fully in the appendix). We may then compute maximum and minimum $\cos ^{\kappa} \theta_{m}, \cos \theta_{i}, \cos \theta_{r}$ over these cones, and estimate error by interval width. The full expression for estimated error over transport is given in the appendix.

\subsection{Clamping and Visibility}

Evaluation of glossy reflectance over three surface areas, as required by the gather iteration, may be difficult, particularly if surface subdivision has been limited by Aeps rather than satisfaction of error bounds, and if $\kappa$, the facet distribution exponent, has high value. In this case we must estimate the integral of a spikey function over a relatively broad area.

Our solution is to band limit the BRDF in a fashion similar to that presented by Amanatides [1]. We employ the cone estimation techniques of the previous section to determine if the BRDF varies significantly over the given patches - if this variance exceeds a set bound, we "roughen" the reflecting surface, lowering $\kappa$ to broaden the resulting reflection over the estimated cones. We then renormalize the resulting blurred function, as described in [1], to

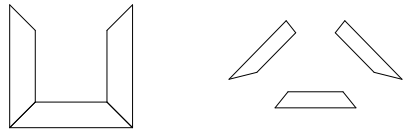

Figure 6: Geometric Configurations

prevent amplification of its low frequency components. We note that the resulting antialiasing is relatively aggressive, significantly dimming or eliminating reflections requiring overmuch blurring.

In implementation, we have computed visibility via jittered ray casting and inheritance similar to that of [7], storing visibility data in interactions as it is computed.

\section{Results}

\subsection{Growth in Transport}

We have measured the growth in transport triples (linked interactions) versus $n$, the maximum number of elements at the finest level of subdivision, over parallel, perpendicular, and "oriented" patches (Figure 7). The corresponding geometries are shown in Figure 6. The graphs show linear or near linear behavior over each range the graph of triples vs. $n$ for the perpendicular case is slightly concave over the lower data points, but subsides to linear with further refinement.

In previous work [7] on hierarchical refinement for radiosity, it was shown that for error estimate proportional to $F_{d j i}$, and sufficient refinement, each subpatch may only interact with other patches in a limited local neighborhood. As discussed in [7], each patch may thus participate in at most $c$ interactions, for some constant $c$ independent of $n$ and $k$. Adaptive refinement thus generates at most $O(n)$ transport interactions. We will show a similar bound for discrete three point transport under glossy reflection.

Recall that the estimate for error in computed transport is proportional to $\Delta F_{j i} S_{k j i}+\Delta S_{k j i} F_{j i}$. Our argument depends on two assumptions:

1. We may bound both $\Delta S_{k j i}$ and $S_{k j i}$ by some $S_{\max }$.

As discussed below, the lower this $S_{\max }$, the smaller the magnitude of the leading coefficient underling the resulting bound.

Note that our argument thus does not apply to perfect specular reflection, as the corresponding BRDF incorporates the Dirac delta function [11]. Equivalently, the argument does not hold over $f_{g}$ for $\kappa=\infty$ (inducing mirror reflection), as we can not provide a finite bound for $S$ in this case.

For finite $\kappa$, however, the desired bound over glossy reflection is achieved by:

$$
\frac{\kappa+2}{8} \max \left(\cos ^{\kappa} \theta_{m}\right) \max \left(\sec \theta_{i}\right) \max \left(\sec \theta_{r}\right)
$$

The maxima over the secant terms are bounded by microfacet selfshadowing.

2. $\Delta F_{j i}$ and $F_{j i}$ within our error estimate are at most proportional to $F_{d j i}$.

Recall that we estimate $F_{j i}$ as $F_{d j i}$, and $\Delta F_{j i}$ as $F_{d j i}^{2}$, thus satisfying this assumption.

Given these assumptions, estimated error is at most proportional to $S_{\max } F_{d j i}$.

We may now show $O(n)$ growth, for sufficient refinement. Consider refinement over interaction $i j$ under an error estimate at worst proportional to $S_{\max } F_{d j i}$. The error estimate is thus proportional to $F_{d j i}$, and therefore, for sufficient refinement, there are at most $O(n)$ such interactions, as discussed in [7].

Consider now an error satisfied link from $i j$ to an interaction $j k$. For sufficient refinement under our subdivision scheme, we may assume that form factors $F_{i j}, F_{j i}, F_{j k}, F_{k j}$ over $p_{i}, p_{j}$, and $p_{k}$ are roughly equal. Furthermore, these satisfying form factors depend only on the error estimate, reflection function, and error bounds, not on $n$ or $k$. 

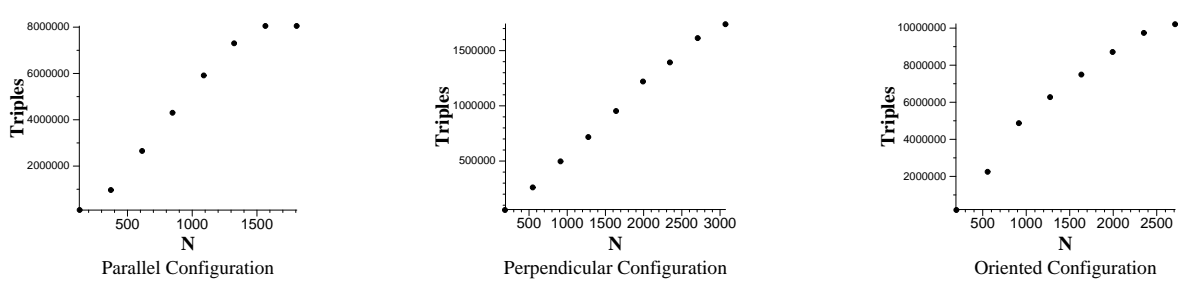

Figure 7: Triples vs. $N$ over Geometry. $\quad$ Error bounds $e=0.1$. Glossy exponent $\kappa=25$. For oriented case $e=0.005$.

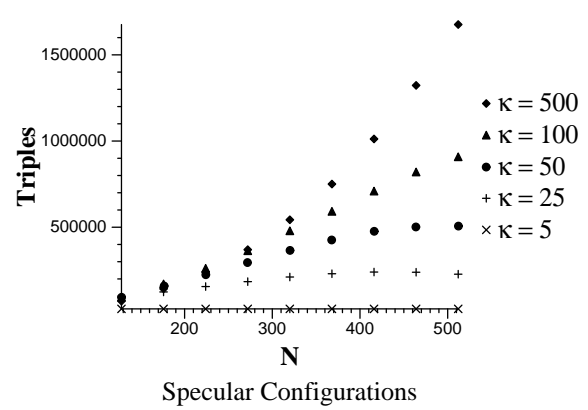

Figure 8: Triples vs. $N$ over $\kappa$. The graph is over parallel polygons for which the error bounds and interpolygon distance have been doubled.

At worst the above form factors are such that $F_{* *} S_{\max }<\mathrm{Eps}$, where Eps is the most restrictive error bound. Note that, as stated above, $F_{* *}$ depends only on the error estimate, reflection function (ie. $S_{\max }$ ), and error bounds. Only some constant number of such form factors may be fitted over the directional hemisphere above $p_{j}$, and thus $i j$ may only be linked to some constant number of interactions $j k$. The total number of linked interactions, and corresponding transport triples, is thus $O(n)$.

Note that the above argument, although it establishes the desired bound, may overstate the potential for links at a given interaction. For a given $i j$, much of the directional reflection into the hemisphere over $p_{j}$ may not achieve $S_{\max }$, and may even be of maximum 0 . That is, the analysis ignores the modulation between the paired error and value terms within the error estimate.

As $\kappa$ increases in magnitude, the corresponding bound $S_{\max }$ must increase as well. We may thus expect greater growth in transport computation with higher specular exponent, as shown in Figure 8. Within this graph, growth is superlinear for $\kappa=500$, though further trials over a higher range of $n=500 \ldots 2000$ have shown that the rate subsides to linear as $n$ increases, allowing sufficient refinement for the local neighborhood property to obtain.

Finally, we note that under specular reflection each element is reflected across every other element perfectly, and to a first approximation is visible from a constant number of other elements in the environment (at least in the case of a convex enclosed room; the analysis is complicated by occlusion and certain worst case alignments). Thus, the number of interactions is at least $O\left(n^{2}\right)-$ we conjecture that it is no worse than this bound.

\subsection{Illumination and Refinement}

Figure 9 shows illumination and meshing over surfaces of varying glossiness (specular exponent). Within each image, the reflecting surface is perpendicular to the diamond shaped light source, and we see the resulting reflection in the direction of the eye. Note the conformation of meshing to the highlight over each surface. The "stretched" nature of the highlight along the axis to the eye is characteristic of Torrance-Sparrow reflection over fairly oblique angles, and accounts for the increased sensitivity of meshing along this axis. The rightmost three images in the figure show the meshing from above. The illumination shown in these images is somewhat unusual - it shows the reflection to the eye as though it had been painted on the reflecting surface, and then viewed from a different location, directly above. The images in Figure 11 show similar

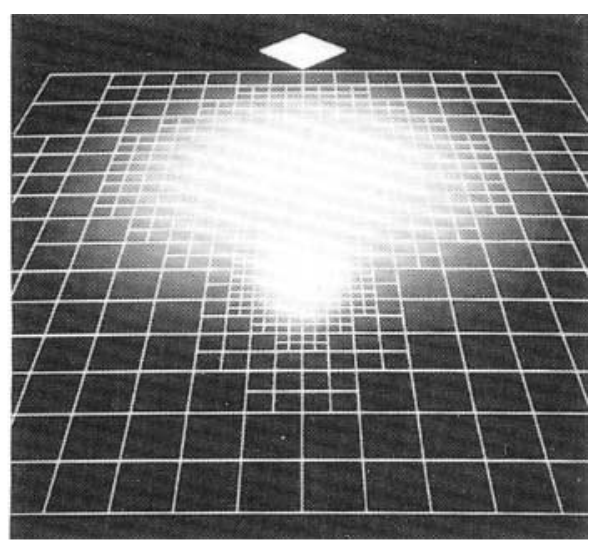

Figure 10: Meshing for glossy and diffuse reflection

\begin{tabular}{|c|c|c|c|c|}
\hline \multicolumn{3}{|l|}{ Figure 9 } & & \\
\hline Max Elements & \multirow{2}{*}{\multicolumn{2}{|c|}{$\begin{array}{r}4160 \\
262144\end{array}$}} & & \\
\hline Max Triples & & & & \\
\hline Computed & \multicolumn{2}{|r|}{$\begin{array}{r}262144 \\
\text { elements }\end{array}$} & triples & time \\
\hline$\kappa=25$ & 790 & 593 & $6706(2.6 \%)$ & $2.2 \mathrm{~s}$ \\
\hline$\kappa=100$ & 1290 & 968 & $24214(9.2 \%)$ & $8.0 \mathrm{~s}$ \\
\hline$\kappa=500$ & 874 & 656 & $12106(4.6 \%)$ & $4.1 \mathrm{~s}$ \\
\hline \multicolumn{3}{|l|}{ Figure 10 } & & \\
\hline Max Elements & \multicolumn{2}{|r|}{16448} & & \\
\hline Max Triples & & & \\
\hline Computed & \multicolumn{2}{|r|}{$\frac{1048576}{\text { elements }}$} & triples & time \\
\hline$\kappa=500$ & 1578 & 1184 & $7834(0.75 \%)$ & $5.0 \mathrm{~s}$ \\
\hline \multicolumn{3}{|l|}{ Figure 12} & & \\
\hline Max Elements & \multirow{2}{*}{\multicolumn{2}{|c|}{$\begin{array}{r}15138 \\
222385209344\end{array}$}} & & \\
\hline Max Triples & & & & \\
\hline Computed & \multicolumn{2}{|c|}{$\begin{array}{cr}222385209344 \\
\text { patches } & \text { elements }\end{array}$} & triples & time \\
\hline$\kappa=500$ & 6479 & 4866 & $70995(0.00003 \%)$ & $3 \mathrm{~m} 13 \mathrm{~s}$ \\
\hline
\end{tabular}

Table 1: Image Statistics

eye/offset views for the reflection of a garish checkerboard.

The image in Figure 10 shows contrasting illumination and meshing induced by diffuse and glossy reflection. Note the distinct meshing for each highlight. Glossy reflection is at a less oblique angle, and thus both the highlight and meshing exhibit less distortion in the direction of the eye.

Note that these scenes are extremely simple - application to more complex environments is still very expensive, despite the employment of hierarchical methods. Motivated by the work of Smits et al. [14] in hierarchical radiosity, we are currently experimenting with importance and radiance weighting over three point transport - preliminary results of this work are shown in Figure 12. The given environment contains four reflectors: the broad face of each of the three "slabs" and the top of the central cube. In addition to the reflections seen in the slabs, note the play of light originating at the lamp at left, reflected off the cube top, and over the upper part of the green wall at right. Total potential transport triples over this environment at the finest level of subdivision is just over 222 billion - our system, under importance and radiance weighting, employs 70,995 , a reduction to 3 hundred-thousandths of 1 percent.

Table 1 provides further statistics for the images. Timings are given for a Silicon Graphics indigo workstation with a single 50 $\mathrm{MHz}$ R4000 processor. The image shown in Figure 12 was generated after seven complete iterations (gathers to all interactions), and total time just over three minutes. 

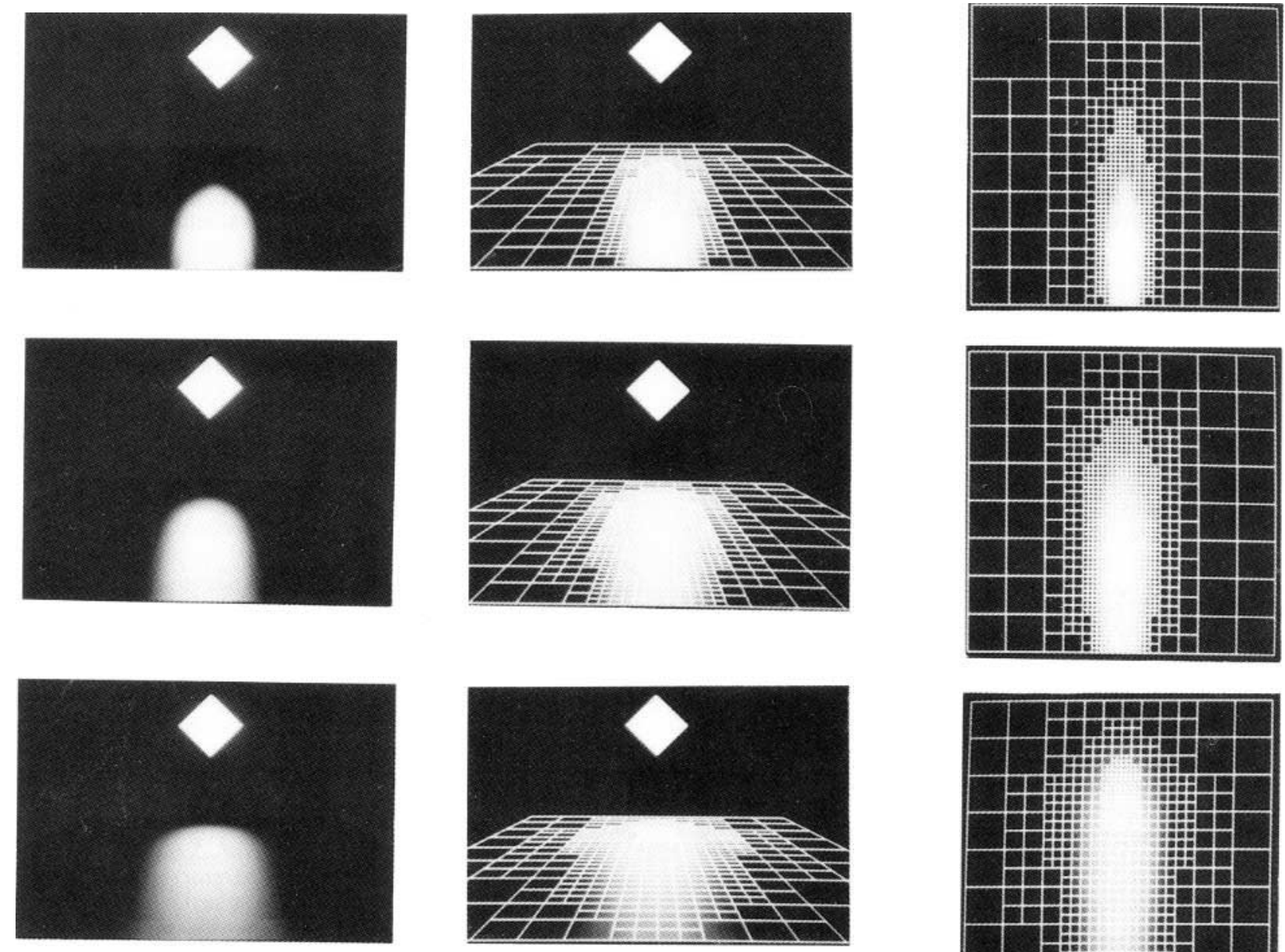

Figure 9: Illumination and Refinement

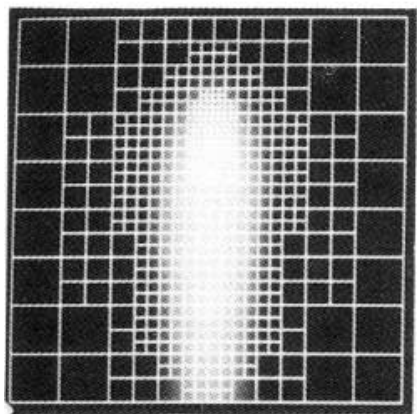

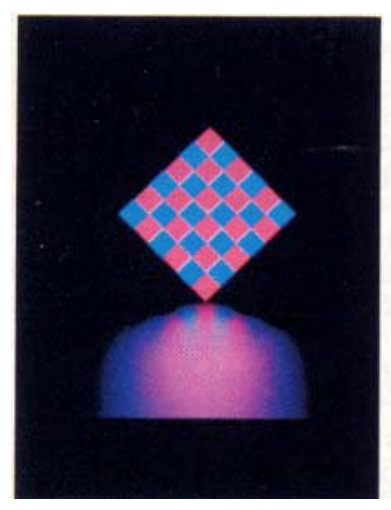

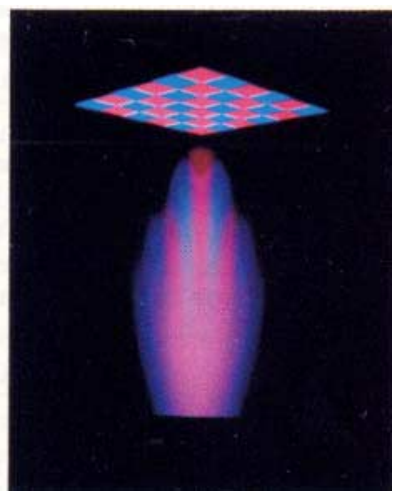

Figure 11: Eye and Offset Views

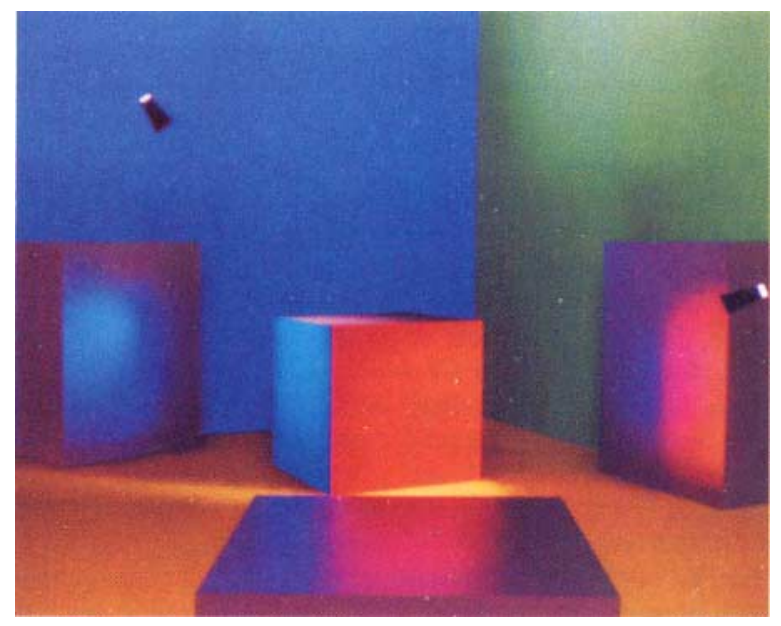

Figure 12: Cube and Slabs 


\section{Discussion}

Recall the matrix formulation shown in Figure 2. For any $n$ of reasonable size, the resulting $n^{3}$ matrix will be unmanageable we have shown, however, that for sufficient refinement the $n^{3}$ entries in the matrix may be approximated to within user specified bounds by $O(n)$ subblocks. The gather and push/pull procedures described in preceding sections allow manipulation and solution over this representation. As discussed in [2], the resulting system may be shown to converge.

Growth in transport is more accurately described as $O\left(n+k^{3}\right)$, where $k$ is the number of input polygonal patches within the environment, as opposed to elements. The $k^{3}$ term is generated by the initial examination of all polygon triples for reflection, and is subsumed by $n$ as the number of elements increases. As the number of polygons in an environment grows, however, the $k^{3}$ term will become prohibitively large. As discussed in [14] with respect to the related problem under hierarchical radiosity, the capability to cluster as well as refine polygons would reduce the difficulty of unnecessary initial interactions. Clustering is arguably the most important open problem in the computation of global illumination.

The hierarchical approach described in this paper was derived by writing the rendering equation in a three point transport formulation. Another option would be to parameterize radiance by position and direction - we believe that a similar hierarchical approach could be employed with the method of discrete ordinates or spherical harmonics.

Finally, we note that, similarly to other algorithms for hierarchical illumination [7, 14], the algorithm described in this paper bounds estimated error over individual transport computations. As discussed in [14], bounding estimated error over individual transport does not easily or necessarily provide a rigorous bound for overall error in the solution. An analysis and means of computing such a bound over hierarchical illumination remains an interesting open problem.

\section{Acknowledgements}

This research was partially supported by equipment grants from Apple and Silicon Graphics Computer Systems and a research grant from the National Science Foundation (CCR 9207966). The authors would like to thank Dr. P. Prusinkiewicz for access to the graphics research facilities at the University of Calgary during the final stages of this work, and Deborah Fowler for her crucial assistance in shooting test images, paste up and much other support and encouragement. Thanks to Cullen Jennings and David Laur for all of their help recording images. We especially thank the anonymous referees for their many helpful comments and suggestions.

\section{References}

[1] Amanatides, J. (1992) Algorithms for the detection and elimination of specular aliasing. Proc. Graphics Interface '92, 86-93.

[2] Aupperle, L. (1993) Hierarchical algorithms for illumination. Doctoral Dissertation, Princeton University.

[3] Blinn, J.F. (1977) Models of light refection for computer synthesized pictures. Computer Graphics 11 (2), 192-198.

[4] Chen, S.E., Rushmeier, H.E., Miller, G., Turner, D. (1991) A progressive multi-pass method for global illumination. Computer Graphics 25 (4), 165-174.

[5] Cook, R.L. (1986) Stochastic sampling in computer graphics. ACM Transactions on Graphics 5 (1), 51-72.

[6] Hall, R. (1989) Illumination and color in computer generated imagery. Springer-Verlag, New York.

[7] Hanrahan, P., Salzman, D., Aupperle, L. (1991) A rapid hierarchical radiosity algorithm. Computer Graphics 25 (4), 197-206.

[8] Immel, D.S., Cohen, M.F., Greenberg, D.P. (1986) A radiosity method for non-diffuse environments. Computer Graphics 20 (4), 133-142.

[9] Kajiya, J.T. (1986) The rendering equation. Computer Graphics $20(4), 143-150$.
[10] Mitchell, D. (1992) Manuscript.

[11] Nicodemus, F.E., Richmond, J.C., Hsia, J.J., Ginsberg, I.W., Limperis, T. (1977) Geometrical considerations and nomenclature for reflectance. National Bureau of Standards monograph, no. 160. [12] Shirley, P. (1990) A ray tracing method for illumination calculation in diffuse-specular scenes. Proc. Graphics Interace '90, 205-212.

[13] Sillion, F.X., Arvo, J.R., Westin, S.H., Greenberg, D.P. (1991) A global illumination solution for general reflectance distributions. Computer Graphics 25 (4), 187-196.

[14] Smits, B.E., Arvo, J.R., Salesin, D.H. (1992) An importancedriven radiosity algorithm. Computer Graphics 26 (2), 273-282.

[15] Torrance, K.E., Sparrow, E.M. (1967) Theory for off-specular reflection from roughened surfaces. J. of the Optical Society of America 57 (9), 1105-1114.

[16] Ward, G.J., Rubinstein, F.M., Clear, R.D. (1988) A ray tracing solution for diffuse environments. Computer Graphics 22 (3), 8592.

\section{Appendix: Error Analysis}

Recall the error expression derived in Section 2.3:

$$
\pi\left(\Delta F_{j i} S_{k j i}+\Delta S_{k j i} F_{j i}\right)
$$

In implementation, we have divided $\Delta S_{k j i}$ into separate components for each subfactor of $f_{g}$. We thus have:

$$
\begin{gathered}
\frac{\kappa+2}{8}\left(\Delta F_{j i} \frac{\cos ^{\kappa} \theta_{m}}{\cos \theta_{i} \cos \theta_{r}}+\Delta \cos ^{\kappa} \theta_{m} F_{j i} \frac{1}{\cos \theta_{i} \cos \theta_{r}}+\right. \\
\left.\Delta \sec \theta_{i} F_{j i} \frac{\cos ^{\kappa} \theta_{m}}{\cos \theta_{r}}+\Delta \sec \theta_{r} F_{j i} \frac{\cos ^{\kappa} \theta_{m}}{\cos \theta_{i}}\right)
\end{gathered}
$$

In implementation, the refinement procedure of Section 3.2 takes an additional argument, Ceps, against which the two estimates of error in reciprocal cosine are tested.

We are left with the computation of $\Delta \sec \theta_{i}, \Delta \sec \theta_{r}$, and $\Delta \cos ^{\kappa} \theta_{m}$. The variance (and associated error) in these cosine terms over given patches $A_{i}, A_{j}, A_{k}$ is determined by the set of possible $\vec{\omega}_{i}, \vec{\omega}_{r}$ lying between the patches (we dispense with' notation in this section).

Consider patches $A_{i}$, and $A_{j}$ (Figure 4): we enclose these patches in spheres $S_{i}, S_{j}$ with centers $c_{i}, c_{j}$, and radii $r_{i}, r_{j}$, respectively. For the moment we will assume that the interiors of $S_{i}$ and $S_{j}$ do not intersect, and thus there exists a tangent cone lying between the spheres.

Note that this cone is a right circular cone centered on the line joining $c_{i}$ and $c_{j}$. Consider the nappe containing $S_{i}$ : it may be regarded as a cone of direction vectors centered about the vector $c_{i}-c_{j}$. We will call this vector cone $C_{i}$. If $p_{i}$ and $p_{j}$ are any two points on or in $S_{i}, S_{j}$, then the vector $p_{i}-p_{j}$ lies within $C_{i} . C_{i}$ thus bounds the set of possible $\vec{\omega}_{i}$. We may characterize $C_{i}$ by the angle $\alpha_{i}$ defined by its axis, $c_{i}-c_{j}$, and boundary - cone $C_{r}$ and angle $\alpha_{r}$ may be similarly defined over $A_{j}$ and $A_{k}$. If either pair of spheres intersect, we set the corresponding $\alpha=\pi$. We may easily compute maxima and minima for $\sec \theta_{i}$ and $\sec \theta_{r}$ given $C_{i}$ and $C_{r}$, and may then compute error in estimation as $(\max -\min ) / 2$.

The cones $C_{i}$ and $C_{r}$ centered about $\vec{\omega}_{i}$ and $\vec{\omega}_{r}$ induce a similar cone of variation about $\vec{\omega}_{m}$ (Figure 5). Application of basic spherical trigonometry yields [2]:

$$
\alpha_{m} \leq \arcsin \min \left(\frac{\sin \left(\alpha_{i} / 2\right)+\sin \left(\alpha_{r} / 2\right)}{\vec{\omega}_{i} \cdot \vec{\omega}_{m}}, 1.0\right)
$$

Given $\alpha_{m}$, determination of $\max \left(\cos ^{\kappa} \theta_{m}\right), \min \left(\cos ^{\kappa} \theta_{m}\right)$, and thus $\Delta \cos ^{\kappa} \theta_{m}$ immediately follows.

Having computed these estimates and maxima, and incorporating the estimates for form factor computation, we may bound and estimate error in transport as:

$$
\begin{gathered}
\frac{\kappa+2}{8}\left(F_{d j i}^{2} \max \left(\cos ^{\kappa} \theta_{m}\right) \max \left(\sec \theta_{i}\right) \max \left(\sec \theta_{r}\right)+\right. \\
\Delta \cos ^{\kappa} \theta_{m} F_{d j i} \max \left(\sec \theta_{i}\right) \max \left(\sec \theta_{r}\right)+ \\
\Delta \sec \theta_{i} F_{d j i} \max \left(\cos ^{\kappa} \theta_{i}\right) \max \left(\sec \theta_{r}\right)+ \\
\Delta \sec \theta_{r} F_{d j i} \max \left(\cos ^{\kappa} \theta_{i}\right) \max \left(\sec \theta_{i}\right)
\end{gathered}
$$

It is this error measure that we employ in our implementation. 